\title{
Síndrome de Di George
}

\author{
Lucía Sierra Santos a,b, Pilar Casaseca García a,c, Alfonso García Moreno a,d \\ y Vicente Martín Gutiérrez a,e
}

a Grupo de Trabajo de Genética

y Enfermedades Raras.

Sociedad Española de Medicina

Familiar y Comunitaria.

${ }^{b}$ Médico de Familia.

Centro de Salud de Manzanares el Real (Consultorio de El Boalo). Madrid (España).

${ }^{c}$ Médico de Familia.

Centro de Salud de

Torrelodones. Madrid (España).

${ }^{d}$ Médico residente de cuarto

año de Medicina de Familia y

Comunitaria. Centor de Salud de

Casteller. Valencia (España).

e Médico de Familia. Centro de

Salud Condado Occidental.

Huelva (España).

Correspondencia:

Lucía Sierra Santos.

C/ Peña Hoyuela, 17.

C.P. 28413 - El Boalo. Madrid

(España).

Correo electrónico:

lusierra@hotmail.com

Recibido el 13 de febrero de 2014.

Aceptado para su publicación el 24 de marzo de 2014

\section{RESUMEN}

El síndrome de Di George o velocardiofacial ocurre en 1:4000 nacidos vivos y es una rara alteración que en el 90 \% de los casos corresponde a una deleción del cromosoma 22 en la porción 22q11. Dicho síndrome puede incluir defectos faciales, cardiopatías congénitas, hipoplasia tímica, hipoparatiroidismo, alteraciones renales, inmunológicas y psiquiátricas. Muchos de estos pacientes pueden llegar a la edad adulta y precisan de atención y seguimiento médico toda su vida.

Palabras clave: Síndrome de DiGeorge. Síndrome Velocardiofacial. Inmunidad. Timo.

\section{ABSTRACT}

\section{DiGeorge syndrome}

The DiGeorge or velocardiofacial syndrome occurs in 1:4000 live births and is a rare condition which corresponds to chromosome 2222 q11 deletion in $90 \%$ of cases. This syndrome may include facial defects, congenital heart disease, thymic hyperplasia, hypoparathyroidism and kidney, immunological and psychiatric disorders. Many of these patients may reach adulthood and require medical care and supervision throughout their whole lives.

Key words: DiGeorge syndrome. Velocardiofacial syndrome. Immunity. Thymus Gland.

\section{INTRODUCCIÓN}

Presentamos el caso de una paciente con síndrome de Di George diagnosticada en la edad adulta. Se trata de un trastorno genético y congénito con una prevalencia de 1 por cada 4.000 nacidos vivos debido a una deleción del brazo corto del cromosoma 22 (22q11), que puede producir hipoparatiroidismo, alteraciones cardiacas e inmunológicas, hipoplasia de timo y alteraciones renales y psiquiátricas ${ }^{1,2}$. Algunos pacientes fallecen en la infancia por complicaciones cardiacas e infecciosas, pero otros pueden llegar a la edad adulta, incluso sin diagnosticar y tener una esperanza de vida prolongada, por lo que los médicos de familia y pediatras deberían estar familiarizados con los síndromes genéticos más frecuentes y ser capaces de tratar y derivar ante la sospecha de los mismos.

El diagnóstico precoz es fundamental para poder tratar y prevenir las posibles complicaciones del síndrome.

\section{OBSERVACIONES CLÍNICAS}

Se trata de una paciente de 36 años que presentaba un retraso mental moderado y brotes psicóticos resistentes al tratamiento. Al ser historiada por primera vez, ya que procedía de otro centro de salud, presentaba múltiples antecedentes que hicieron sospechar que podría tratarse de un síndrome 
genético, por lo que fue derivada al Servicio de Genética Clínica de su hospital de referencia.

Nuestra paciente era la tercera hija de padres sanos no consanguíneos y tenía un primo hermano materno con retraso mental no filiado sin cardiopatía asociada. Nació tras un embarazo normal y un parto eutócico a las 36 semanas de gestación. Pesó 1.900 gramos al nacer (percentil 25-50) y precisó ingreso en la unidad neonatal durante 25 días por dificultad en la deglución, ya que presentaba un paladar blando hendido. Fue intervenida de este defecto a los 14 meses de vida. A los 9 años fue operada de una comunicación interauricular sin otras anomalías cardiacas asociadas. Fue intervenida, además, a los 20 años de un colesteatoma, insuficiencia venosa crónica y un fibroma de mama. Su desarrollo psicomotor fue lento y tuvo un rendimiento escolar pobre. No acabó el graduado escolar y le fue concedida una minusvalía del $37 \%$.

A la exploración destacaba una obesidad troncular y unos rasgos faciales particulares (discreta hipoplasia malar, hendiduras palpebrales estrechas, nariz con puente ancho y aspecto tubular y boca pequeña). El paladar estaba íntegro (corregido), la úvula bífida, las orejas eran pequeñas con implantación normal pero presentaban un sobreplegamiento del hélix. La voz que tenía la paciente era nasal y el cuello corto. Los dedos de las manos eran afilados y pequeños.

Ante la sospecha del Síndrome de Di George, se realizaron en Genética estudios moleculares específicos, que dieron como resultado una deleción de $3 \mathrm{Mb}$ en la región cromosómica 22q11.2, confirmándose así el diagnóstico esperado.

Los padres de la paciente fueron sometidos a un estudio citogenético molecular para descartar la presencia de reestructuración en balance de alguno de ellos y fue descartado este extremo. Tampoco su primo presentó esta alteración.

Tras el diagnóstico, se completó el estudio de la paciente con una ecografía abdominopélvica, que resultó ser normal, y con estudio analítico e inmunológico. Se evidenció un hipotiroidismo subclínico y un hipoparatiroidismo: TSH 9,5 uUl/ml (0,25-4,94); T4 normal; PTH 10 pg/ml (12-65); calcio, en el límite bajo de la normalidad, $8,8 \mathrm{mg} / \mathrm{ml}(8,8-10,6)$.

En el estudio inmunológico presentaba una disminución porcentual de los linfocitos CD3 con un aumento de los linfocitos Natural Killers. No tenía, por lo tanto, una linfopenia absoluta.
La paciente precisó tratamiento psiquiátrico y baja médica durante varias semanas por el brote psicótico, que finalmente fue controlado. En la actualidad realiza un trabajo remunerado adaptado a sus capacidades y continúa con sus revisiones periódicas.

\section{COMENTARIOS}

Desde que en 1968 el pediatra estadounidendese Angelo Di George describió dicho síndrome, se han publicado múltiples casos y se conoce que es la segunda causa de defectos cardiacos congénitos, después del Síndrome de Down ${ }^{3}$.

Es también conocido como Síndrome Velocardiofacial, debido a un defecto incompleto del desarrollo de los arcos braquiales ${ }^{2}$ que puede producir hipoparatiroidismo con hipocalcemia; hipoplasia tímica con trastorno de la inmunidad celular, lo que propicia infecciones frecuentes; cardiopatías conotroncales y malformaciones faciales, como orejas pequeñas, hendiduras palpebrales pequeñas, labio leporino o paladar hendido; trastornos renales y trastornos psiquiátricos en relación con la dopamina plasmática, dependiente de la encima catecolO-metiltransferasa (COMT) cuyo gen se localiza en la región $22 \mathrm{q} 11^{2,4,5}$.

En 1981, de la Chapelle publicó el defecto congénito concreto subyacente. El $90 \%$ de los casos de Síndrome de Di George se produce por microdeleción (pérdida de una porción) del brazo corto del cromosoma 22, región q11. En un $10 \%$ de los casos no se encuentra dicha deleción, sugiriendo una heterogenicidad genética y la existencia de otros loci que determinan el mismo fenotipo. Además, no existe relación entre el tamaño de la deleción y la penetrancia clínica ${ }^{6-8}$.

El Síndrome de Di George ocurre en uno de cada 4.000 nacidos vivos, y el $90 \%$ de los casos son esporádicos. En un $10 \%$ de las familias con un caso de Síndrome de Di George existe un origen hereditario y se comporta de forma autosómica dominante, por lo que una persona que padezca el problema tiene el $50 \%$ de posibilidades de transmitirla a cada uno de sus hijos $2,9,10$.

Al encontrar un cuadro clínico sugerente de Síndrome de Di George hay que realizar: un estudio analítico e inmunológico, ecografía abdominal para descartar alteraciones renales, ecocardiografía y estudios de hibridación in situ fluorescentes (FISH) 
diseñado para detectar pequeños grupos de genes eliminados en la región 22q11. Los padres de los niños afectos han de ser valorados mediante dicha técnica para descartar que se trate de un problema transmisible y no esporádico. Un pequeño porcentaje de niños afectados, con grandes deficiencias inmunológicas o cardiacas no sobreviven al primer año de vida, pero la mayoría sobrepasan esta edad y llegarán a adultos, por lo que no es extraño que los médicos de familia y pediatras de Atención Primaria tengamos pacientes con este síndrome sin diagnosticar.

El diagnóstico es importante para poder valorar y atender adecuadamente sus múltiples problemas y poder prever las complicaciones futuras, poner tratamiento precoz a las infecciones y detectar tempranamente los problemas psiquiátricos que debuten ${ }^{11}$. Asimismo, es prioritario para el asesoramiento genético.

El seguimiento, acompañamiento, información y coordinación de dichos pacientes corresponde al médico de familia, ya que con el tiempo precisan múltiples especialistas que atiendan sus necesidades médicas y quirúrgicas.

\section{BIBLIOGRAFÍA}

1. McDonald-McGinn DM, Sullivan KE. Chromosome 22q11.2 deletion syndrome (DiGeorge syndrome/velocardiofacial syndrome). Medicine (Baltimore). 2011; 90 (1): 1-18.
2. Aglony M, Lizama M, Méndez C, Navarrete C, Garay F, Repetto $\mathrm{G}$ et al. Manifestaciones clínicas y variabilidad inmunológica en nueve pacientes con síndrome de Di George. Rev Med Chile 2004; 132 (1): 26-32.

3. DiGeorge AM. Congenital absence of the thymus and its immunologic consequences: concurrence with congenital hypoparathyroidism. White Plains, NY: March of DimesBirth Defects Foundation 1968:116-21 (abstract).

4. Momma K. Cardiovascular anomalies associated with chromosome 22q11.2 deletion syndrome. Am J Cardiol 2010; 105 (11): 1617-24.

5. Jolin EM, Weller RA, Weller EB. Psychosis in children with velocardiofacial syndrome (22q11.2 deletion syndrome). Curr Psychiatry Rep. 2009; 11 (2): 99-105.

6. Desmaze C, Prieur M, Amblard F, Aikem M, LeDeist F, Demczuk $S$ et al. Physical mapping by FISH of the DiGeorge critical region (DGCR): involvement of the region in familial cases. Am J Hum Genet. 1993; 53 (6): 1239-49.

7. De la Chapelle A, Herva R, Koivisto M, Aula P. A deletion in chromosome 22 can cause DiGeorge syndrome. Hum Genet. 1981; 57 (3): 253-6.

8. Vera E, Salado C, Botella MP, Rodríguez A, Díez I, Gamarra A. Síndrome de DiGeorge asociado a hemimelia. An Pediatr. 2007; 66 (6): 628-30.

9. Muñoz S, Garay F, Flores I, Heusser F, Talesnik E, Aracena $M$ et al. Heterogeneidad de la presentación clínica del síndrome de microdeleción del cromosoma 22, región q11. Rev Med Chile. 2001; 129 (5): 515-21.

10. Oskarsdottir S, Vujic M, Fasth A. Incidence and prevalence of 22q11 deletion syndrome: a population based study in Western Sweden. Arch Dis Child. 2004; 89 (2): 148-51.

11. Ousley OY, Smearman E, Fernandez-Carriba S, Rockers $\mathrm{KA}$, Coleman K, Walker EF et al. Axis I psychiatric diagnoses in adolescents and young adults with 22q11 deletion syndrome. Eur Psychiatry. 2013; 28 (7): 417-22. 